\title{
Improved Fracture Models for Relocation Modeling
}

\section{L3:FMC.FUEL.P15.11}

W. Jiang, INL

B. W. Spencer, INL

D. Schwen, INL

K. A. Gamble, INL

L. Liu, Utah State University

January 30, 2018 


\section{REVISION LOG}

\begin{tabular}{|c|c|c|l|}
\hline $\begin{array}{c}\text { Revisio } \\
\mathbf{n}\end{array}$ & Date & $\begin{array}{c}\text { Affected } \\
\text { Pages }\end{array}$ & \multicolumn{1}{c|}{ Revision Description } \\
\hline 0000 & $1 / 30 / 2018$ & All & Initial Release \\
\hline & & & \\
\hline & & & \\
\hline & & & \\
\hline
\end{tabular}

Document pages that are:

Export Controlled

IP/Proprietary/NDA

Controlled

Sensitive Controlled

This report was prepared as an account of work sponsored by an agency of the United States Government. Neither the United States Government nor any agency thereof, nor any of their employees, makes any warranty, express or implied, or assumes any legal liability or responsibility for the accuracy, completeness, or usefulness of any information, apparatus, product, or process disclosed, or represents that its use would not infringe privately owned rights. Reference herein to any specific commercial product, process, or service by trade name, trademark, manufacturer, or otherwise, does not necessarily constitute or imply its endorsement, recommendation, or favoring by the United States Government or any agency thereof. The views and opinions of authors expressed herein do not necessarily state or reflect those of the United States Government or any agency thereof.

\section{Requested Distribution:}

To:

Copy: 


\title{
Improved Fracture Models for Relocation Modeling
}

\author{
W. Jiang ${ }^{1}$, B. W. Spencer ${ }^{1}$, D. Schwen ${ }^{1}$, K. A. Gamble ${ }^{1}$, L. Liu ${ }^{2}$ \\ ${ }^{1}$ Fuels Modeling and Simulation \\ Idaho National Laboratory \\ P.O. Box 1625 \\ Idaho Falls, ID 83415-3840 \\ ${ }^{2}$ Mechanical \& Aerospace Engineering Department \\ Utah State University \\ Logan, UT 84322-4130
}

January 30, 2018

\section{Introduction}

The BISON nuclear fuel performance code is being developed to provide a modern tool that has the flexibility to analyze a wide variety of fuel forms and to model conditions and phenomena that could not be represented in legacy tools. There are a number of motivations for this, including providing support for development of advanced fuel with improved accident tolerance for existing light water (LWR) reactors, improved understanding of mechanisms in fuel designs in current use in a wider variety of conditions, and facilitating the development of fuel for advanced reactor designs.

To accomplish these goals, it is clear that BISON must rely on models of fuel behavior that are based on fundamental physical behavior, rather than on empirical correlations that represent that behavior in a simplified fashion. BISON still does employ many empirical models that were originally developed for other fuel performance codes, but efforts are underway to replace these with models that are more physically based.

Radial relocation in LWR fuel is an example of a phenomenon that is currently represented by an empirical model, but which is ripe for replacement by physically based models. During normal operation, ceramic LWR fuel experiences significant fracturing that is driven by spatially nonuniform volumetric expansion. This occurs due to the significant thermal gradients that occur within both fresh and irradiated fuel, as well as nonuniform swelling due to fission products that occurs over longer-term irradiation. Fracture and fragmentation of fuel allows the outer radius of the fuel pellet to expand due to the loss of mechanical constraint and outward radial migration of fragments. This radial expansion has a significant effect on the fuel system response because it decreases the size of the gap between the fuel and cladding. This decreases the thermal resistance across that gap, which leads to decreased fuel centerline temperatures.

In practice, radial relocation is typically modeled using empirical models that are tuned to fit experimental data. The model currently used by BISON is based on the model in the ESCORE code [1], with parameters that have been tuned to better match a database of experimental results [2]. Because the underlying mechanisms for relocation are largely due to fracture, an CASL-supported effort was previously undertaken to assess the ability of several fracture mechanics methods to represent relocation [3] during an initial ramp to power on a fresh LWR fuel rod. Figure 1 shows a comparison of the radial relocation predicted by the models assessed in that study.

The three fracture modeling approaches considered in that study all predicted radial relocation that was significantly lower than the relocation predicted by the empirical relocation model that has been calibrated to experimental data. 


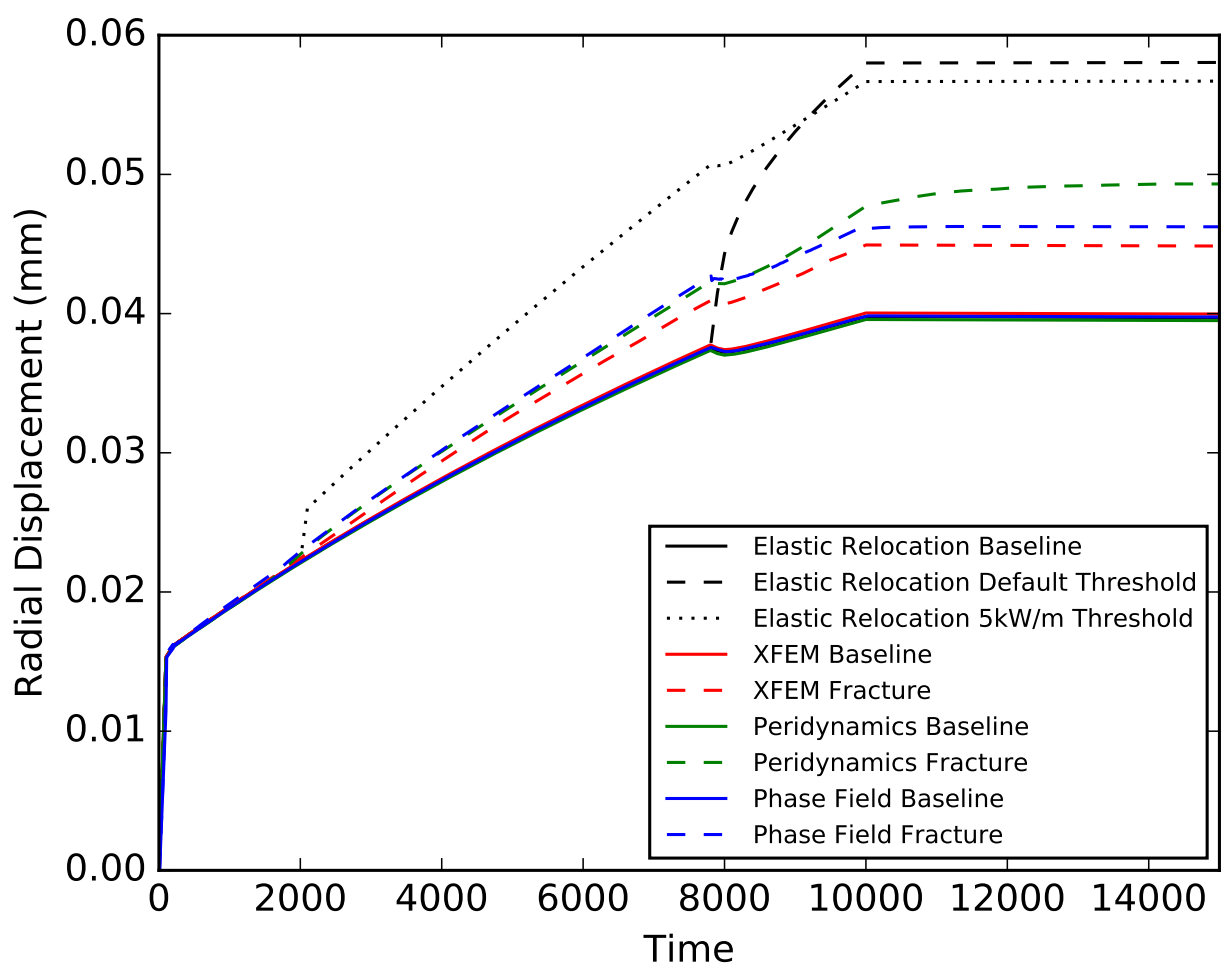

Figure 1: Comparison of radial displacement predicted in fresh fuel in a prototypical pressurized water reactor fuel rod during an initial rise to full power $(25 \mathrm{~kW} / \mathrm{m})$ over $10000 \mathrm{~s}$. Results are compared using three fracture modeling approaches: XFEM, peridynamics, and phase field. For all fracture approaches, a baseline result with fracture disabled is shown. These are also compared against a model with elastic fuel using BISON's standard radial relocation model with two sets of parameters, as well as a baseline elastic model without relocation enabled.

It is believed that a major reason for this underprediction is that the fracture models employed in that study did not include the effect of crack residual opening. During typical operation, the power will be cycled over time, and cracks will open and re-close. However, roughness of the crack surface will prevent the cracks from completely re-closing, which is expected to lead to circumferential cracking. This is expected to significantly increase the amount of radial relocation compared to what would be predicted by a model that only includes radial cracking.

The work reported here builds on that previous work to include the effects of residual opening and circumferential cracking. While multiple modeling approaches were pursued in the previous work, the present work focused exclusively on the extended finite element method (XFEM). Of the methods previously studied, XFEM is likely the most applicable to engineering-scale fuel analysis because it can be used on relatively coarse meshes with computational costs comparable to other engineering-scale fuel models. This report documents the following work:

- XFEM Model Development Several major developments have been made to the XFEM algorithms in MOOSE/BISON in support of this work. This includes work to support the use of interface models with stateful material data, which allows for cohesive zone models and models that include residual crack opening to represent mechanical interactions across cracks. The code architecture for evaluating fracture integrals as criteria for fracture growth is also in the process of a significant re-design to share common code with the fracture integral evaluations for stationary cracks in MOOSE. Improvements have been made to the treatment of elements cut by XFEM on free surfaces where contact or other boundary conditions are applied to permit nucleation and propagation of cracks with XFEM in models that include both fuel and cladding.

- Application to Modeling Radial Relocation in LWR Fuel Under Power Cycles The improved capabilities have been applied to demonstrate the effect of including residual crack opening in fracturing LWR fuel where the cracks are represented using XFEM. This model includes both fuel and cladding, which is a significant 
improvement over previous models. In addition, it includes a model for mechanical interface behavior that represents residual opening due to crack roughness. This is demonstrated to result in increased relocation over multiple power cycles and also drives cracks to propagate in a circumferential orientation.

The details of the developments in these areas are described in corresponding sections of this report. 


\section{XFEM Model Development}

\subsection{Support for interface models with stateful material properties}

In MOOSE, the Material system stores stateful material properties on standard quadrature points. This system permits dependencies of one material model on another, which permits models for various aspects of material behavior to be used interchangeably. It is desired to use a similar system for mechanical (or other) constraints across opposing crack surfaces in XFEM, but the Material system in MOOSE does not allow for the storage of stateful properties at locations other than the standard integration points.

To enforce cohesive-like constraints along XFEM crack surfaces, stateful material properties need to be stored on non-standard quadrature points. For this purpose, a new XFEMMaterialManager class based on UserObject has been implemented to manage the stateful material properties at the quadrature points along the crack surface. The application programming interface (API) of this class has been designed to match that of the standard material system, so functions like getMaterialProperty that are used with the standard material system to get references to material properties behave the same as they do for standard materials.

With this new capability, cohesive zone methods (CZM) can be used in conjunction with XFEM to represent tractionseparation behavior after cracks are introduced into the model. In a typical CZM, as the crack surfaces separate, the interface behavior is governed by a penalty formulation in which tractions increase until a maximum value (the material's tensile strength) is reached. The cohesive model then switches to a softening behavior, in which increasing opening results in decreasing traction until it drops to zero, at which point the material has lost all its strength. Implementing such a model requires storing stateful properties. For the most basic such model, the maximum opening needs to be stored as a stateful material property. To demonstrate this capability, a simple bi-linear cohesive law has been developed based on XFEMMaterialManagerConstraint and applied on a XFEM cut crack surface (see Figure 2(a)). The measured traction and separation curve at crack surface is shown in Figure 2(b), which is exactly the same behavior as is prescribed in the cohesive law.

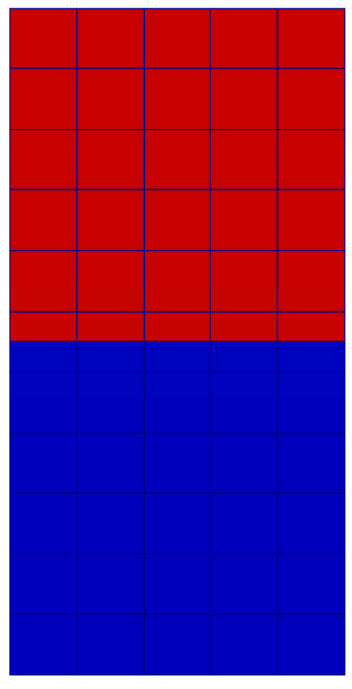

(a) A block is cut by XFEM

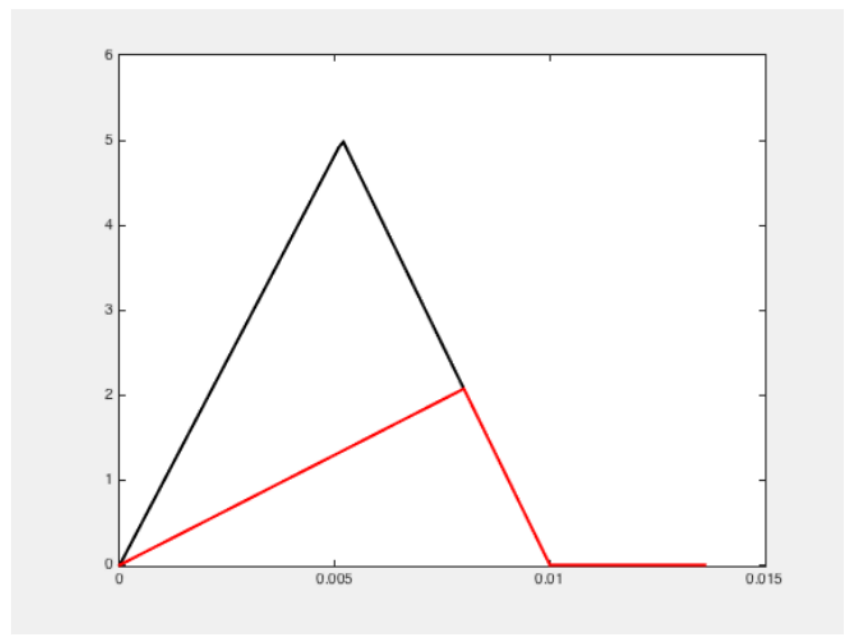

(b) The traction separation curve along crack surface

Figure 2: A CZM demonstration.

For the current study, a fracture mechanics-based criterion is used for crack propagation, so it is assumed that when an XFEM crack has been extended into a new element, there is no remaining cohesive strength. The interface model developed for this purpose treats the crack is being traction-free under tensile loading, but prevents interpenetration of the crack surfaces beyond a residual amount in compression. This model requires the use of a stateful material property, which is managed by the system described above. 


\subsection{Crack propagation criteria based on mixed-mode stress intensity factors}

Under general mixed-mode loading, the asymptotic near tip hoop stress takes the following form:

$$
\sigma_{\theta \theta}=\frac{K_{I}}{4 \sqrt{2 \pi r}}[3 \cos (\theta / 2)+\cos (3 \theta / 2)]+\frac{K_{I I}}{4 \sqrt{2 \pi r}}[-3 \sin (\theta / 2)-3 \sin (3 \theta / 2)]
$$

where $K_{I}$ and $K_{I I}$ are the Mode-I and Mode-II stress intensity factors, respectively.

To determine the direction of crack propagation, the maximum hoop stress criterion is used, which states that the crack will propagate from its tip in a direction $\theta_{c}$ so that $\sigma_{\theta \theta}$ is maximum. The roots of the equation for $\theta_{c}$ are given as

$$
\theta_{c}=2 \arctan \frac{1}{4}\left(\frac{K_{I}}{K_{I I}} \pm \sqrt{\left(\frac{K_{I}}{K_{I I}}\right)^{2}+8}\right)
$$

The correct root of $\theta_{c}$ is chosen as the one which gives larger hoop stress given in Equation 1. The stress intensity factors are computed using domain forms of the interaction integrals.

For some time, a capability has existed in MOOSE to evaluate interaction integrals to obtain the mixed-mode stress intensity factors $K_{I}$ and $K_{I} I$. This was originally developed for mesh-conforming cracks [4], and was later modified to permit use with XFEM [5]. The development of this capability was motivated by the need to assess the probability of fracture initiation at the locations of flaws in embrittled reactor pressure vessels for the Department of Energy's Light Water Reactor Sustainability (LWRS) program. For that application, it is sufficient to compute these properties for stationary cracks, and the initial implementation was restricted to those cases.

A prototype implementation of an ability to calculate fracture integrals about crack tips that are potentially propagating through the mesh for use as a growth criterion with XFEM was previously developed, but to avoid code duplication, it is desirable to have a single code implementation of fracture integrals that is used for both stationary and propagating cracks. A number of important architectural changes were made to the fracture integral capability in MOOSE to permit the location of the crack tip and the number of points along crack tips to change during an analysis. The fracture integrals no longer make use of auxiliary variables for computing quantities used in their calculations because the number of those variables is fixed to the number of crack points, and cannot change during the analysis. The code that computes fracture integrals has been changed to be based on a VectorPostprocessor, rather than a set of Postprocessor objects, also to allow for a changing number of points. This system has also been migrated from the SolidMechanics MOOSE module to the TensorMechanics module, as that is where all future MOOSE mechanics model development will take place. Work is currently underway to allow the XFEM system to make use of the fracture integrals computed by this modified system to drive propagation of cracks, which will eliminate the need for a separate implementation of those integrals within XFEM.

\subsection{Improved support for use of XFEM with models that include fuel and cladding}

The initial implementation of XFEM in MOOSE focused exclusively on the handling of volume integrals on cut elements, and did not properly treat the integration over surfaces of cut elements. Early demonstrations of this capability only included the fuel, without representing the fuel/cladding gap or the cladding directly in the model. Temperatures were prescribed as Dirichlet boundary conditions to the outer surface of the fuel, and the time history of that prescribed temperature was obtained from a separate BISON model of a full fuel/cladding system without fracture. Although this approach permitted a simplified representation of fracture, it was inconvenient because it required running two separate models. It was also potentially inaccurate, as the fuel/cladding model did not include the effects of fracture on the gap size.

Later development of XFEM permitted its use with thermal and mechanical contact with a small number of initially prescribed cracks, but there were accuracy issues with integrated boundary conditions (such as the plenum pressure) applied to the cracked fuel surface. Pressures applied to a cracked surface were over-represented, and computation of plenum volume was inaccurate. These inaccuracies are relatively minor with a small number of cracks, but can be 
significant if there are many cracks intersecting the outer surface of the fuel. This issue has been addressed to permit the use of a model with fuel and cladding in the present study.

In the XFEM implementation in MOOSE, for volume integration, the weights of the standard Gauss integration points are modified to permit integration of quantities over partial elements. The new weights are obtained either by taking the volume fraction of the portion of a cut element on one side of an interface, or by using a moment-fitting approach [6]. In the present work, the volume fraction approach has been extended to permit its use with boundary integrations by using the fraction of the surface area of an element on one side of a cut as a multiplier for the surface integration point weights. The physical portion of the cut element side (shown in red in Figure 3) is obtained from the XFEM algorithm's information about the element fragment. This is implemented for both 2D and 3D cases in MOOSE.
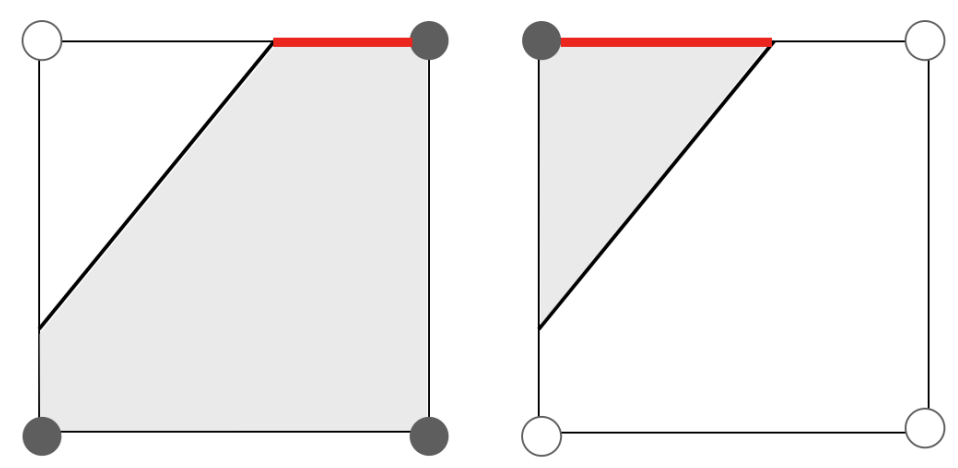

Figure 3: Boundary integration along XFEM cut element side

The improvement in accuracy from this development is demonstrated on a simple diffusion problem, in which a crack propagating from the middle of the top surface of a rectangular domain to its center is added and a constant flux boundary condition is applied at the top surface. Figure 4 shows contours of the concentration field both without and with the adjustment made to the surface integration weights. The adjustment of the integration weights allows the flux boundary condition to be accurately enforced and results in the correct solution, in which the concentration field is constant in the horizontal direction at a given vertical location.

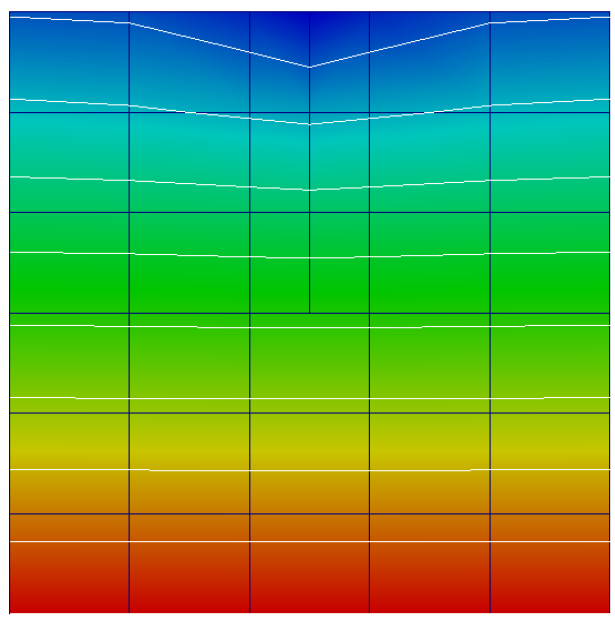

(a) Without adjustment for the surface integration weights

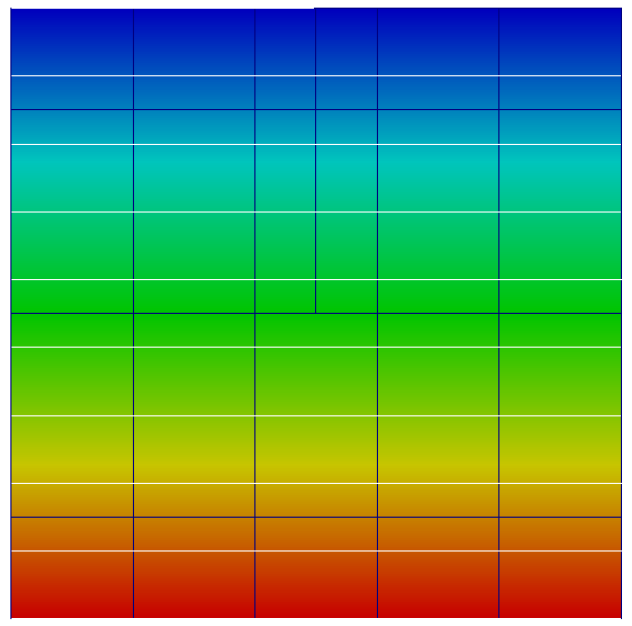

(b) With adjustment for the surface integration weights

Figure 4: Demonstration of improved integration of surface integrals on elements cut by XFEM on a simple diffusion problem with a constant flux boundary condition applied at the top surface. Contours of the concentration field are shown. 


\section{Application to Modeling Radial Relocation in LWR Fuel Under Power Cy- cles}

To assess the effects of including a surface interaction model with XFEM on radial relocation in LWR fuel, a 2D planar cross section of an LWR fuel/cladding system was modeled. This model includes the thermal and mechanical behavior in the fuel and cladding materials, as well as the thermal contact between the fuel and cladding. The fuel pellet has an outer radius of $0.41 \mathrm{~cm}$. The cladding inner radius is $0.418 \mathrm{~cm}$ and the cladding outer radius is $0.475 \mathrm{~cm}$. The simulation assumed a Young's modulus of $2 \times 10^{11} \mathrm{~Pa}$, a Poisson's ratio of 0.345 , a thermal expansion coefficient of $10 \times 10^{6} K^{-1}$, and a constant thermal conductivity of $5.0 \mathrm{~W} /(\mathrm{mK})$ for the fuel. To demonstrate the effect of including residual crack opening in the model over multiple power cycles, the power was ramped up from zero to a linear power of $25 \mathrm{~kW} / \mathrm{m}$ over $10000 \mathrm{~s}$, and then ramped back down to zero over the next $10000 \mathrm{~s}$. This same power cycle was then repeated. At the beginning of the analysis, the coolant temperature was increased from an initial temperature of $298 \mathrm{~K}$ to $580 \mathrm{~K}$ (hot zero power), and then kept at that level through the rest of the simulation.

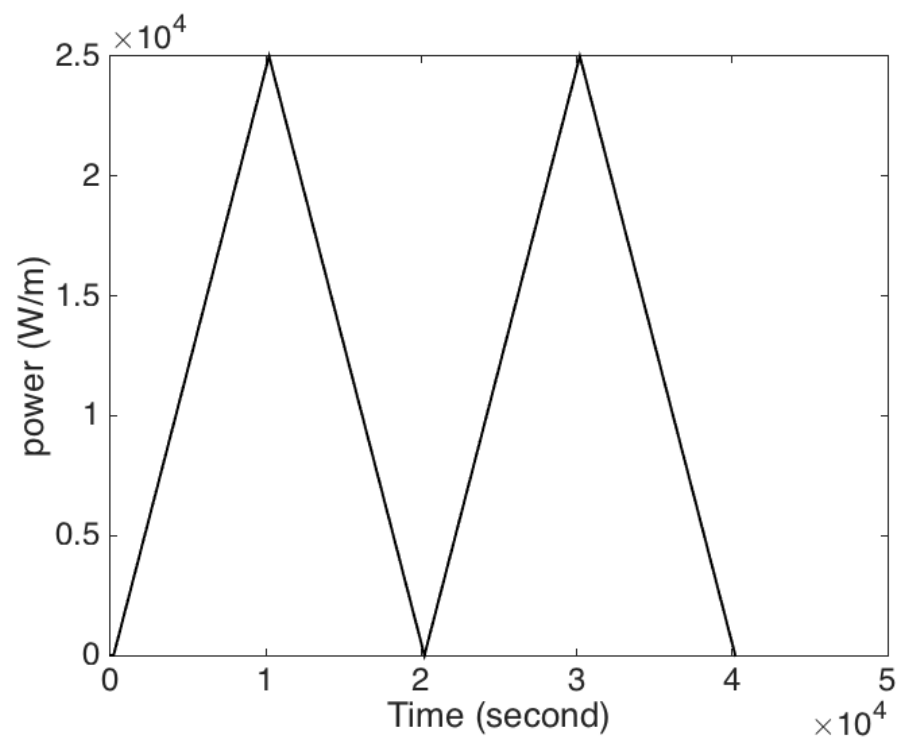

Figure 5: Power history

In the XFEM model, cracks were permitted to initiate in elements adjacent to the exterior boundary of the fuel pellet, and extend from existing cracks in adjacent elements. Cracks are initiated if the maximum hoop stress exceeds $80 \mathrm{MPa}$ and they are grown if the effective $\mathrm{K}$ exceeds $2.5 \mathrm{MPa} \sqrt{\mathrm{m}}$. Whenever a crack meets the criterion for growth, its length is incrementally extended in that time step by a constant $\Delta a=0.00025 \mathrm{~m}$.

When a crack closes, it cannot go back to the original position due to the roughness of the newly created surface. In this study, we used the following simple residual opening model:

$$
\text { Residual opening }= \begin{cases}\frac{1}{2} \text { Maximum opening, } & \text { if Maximum opening }<=\text { Fuel roughness } \\ \frac{1}{2} \text { Fuel roughness, } & \text { if Maximum opening }>\text { Fuel roughness }\end{cases}
$$

The crack roughness was assumed to be $5.0 \times 10^{-5} \mathrm{~m}$ and the maximum opening is a stateful material property stored by the XFEMMaterialManager. This initial model is quite simple, but adequate to assess the impact of residual opening on the response. It also demonstrates the use of the new framework for managing material properties on interfaces. This system can be readily extended to develop more advanced interface models based on experimental observations that could include a number of phenomena, including healing effects.

Figure 6 shows the finite element mesh used for the XFEM fuel pellet and cladding simulation. The temperature fields on the fractured domain using the residual opening model are shown in Figure 7. As can be seen, the cracks propagate 
inward radially as the power increases. When the power decreases, the residual opening model prevents the crack from closing back fully. This causes tensile stress in the circumferential direction and thus some of the cracks propagate and turn to the circumferential direction. It should be noted that the temperatures were constrained to be equal on opposing sides of the XFEM cracks. In the future, the realism of this model can be improved by including a model for gap conductance similar to that used for fuel/cladding gaps.

To highlight the effects of the residual opening model, the same model was also run without the residual opening model for comparison. As can be seen in Figure 8, the cracks completely close when the power is ramped down, and there is no further crack propagation after the end of the initial power ramp.

Figure 9 shows a comparison of the time history of the average radial displacement on the outer surface of the fuel pellet predicted with and without the residual opening model. For reference, it also shows a baseline plot of the radial displacement without any fracture model. The radial displacement is due both to the thermal expansion and the formation of the cracks. The effect of cracks can be seen by comparing the displacements from the fracture models with those from the baseline case. It is important to note that the current model employs a generalized plane strain condition, which results in reduced radial displacment relative to the results of previous work shown in Figure 1, which used plane strain conditions because the generalized plane strain capability was not sufficiently mature at that time.

The radial displacement during the first power ramp is unaffected by the residual opening model. However, its effects are very pronounced as the power is ramped down, as the displacement returns to a higher residual value that it does without the residual opening model. The peak displacement in the second power ramp is noticeably higher than during the first power ramp. Although our residual opening model is rather simple, this indicates that it can account for crack closing effects seen during power cycles. There is clearly more work to do here to validate this model against experimental data, but this demonstrates that the model can capture this mechanism.

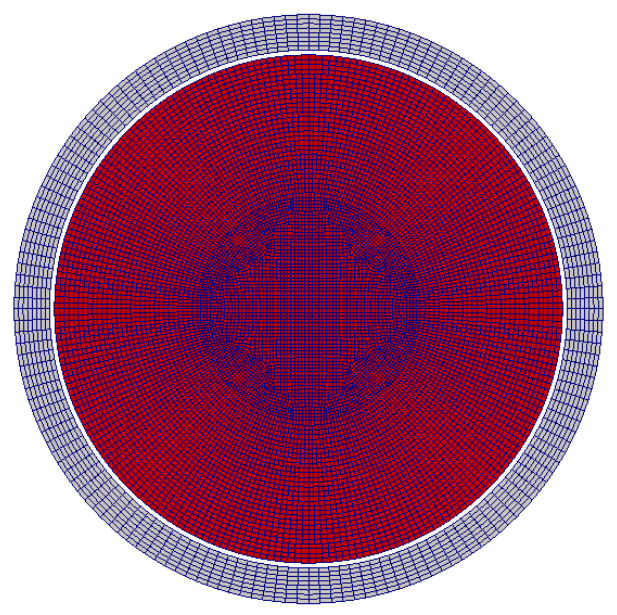

Figure 6: Mesh used for the XFEM simulation, including pellet and cladding. 


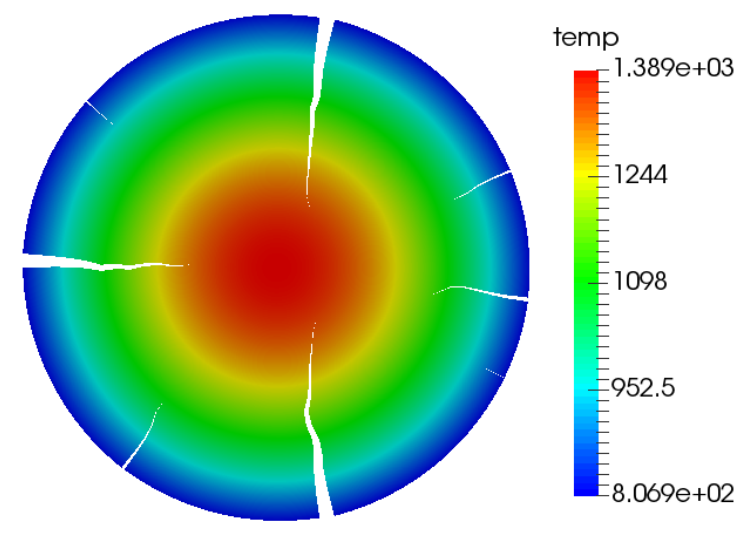

(a) $\mathrm{t}=10000 \mathrm{~s}$

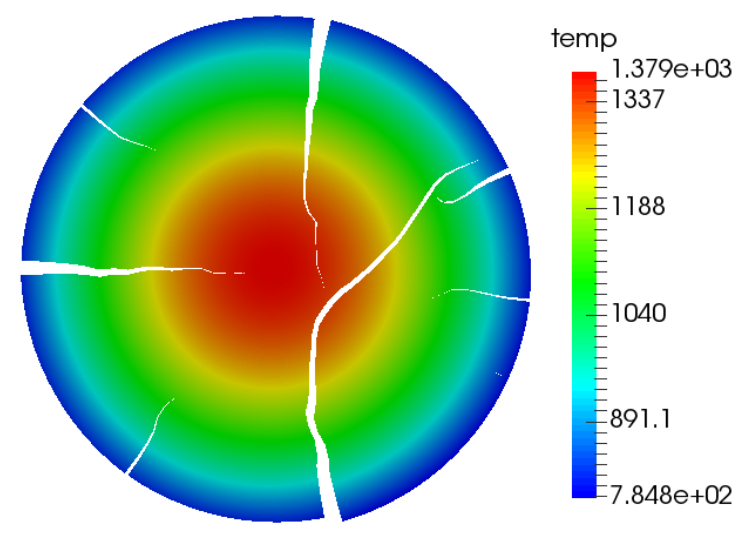

(c) $\mathrm{t}=30000 \mathrm{~s}$

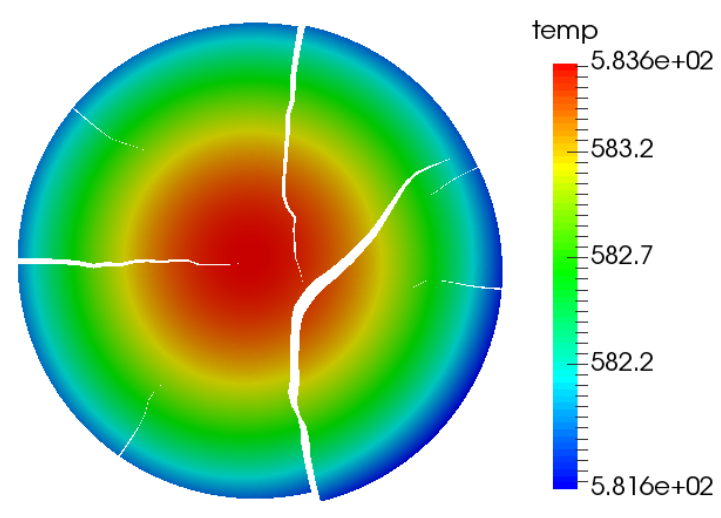

(b) $t=20000 \mathrm{~s}$

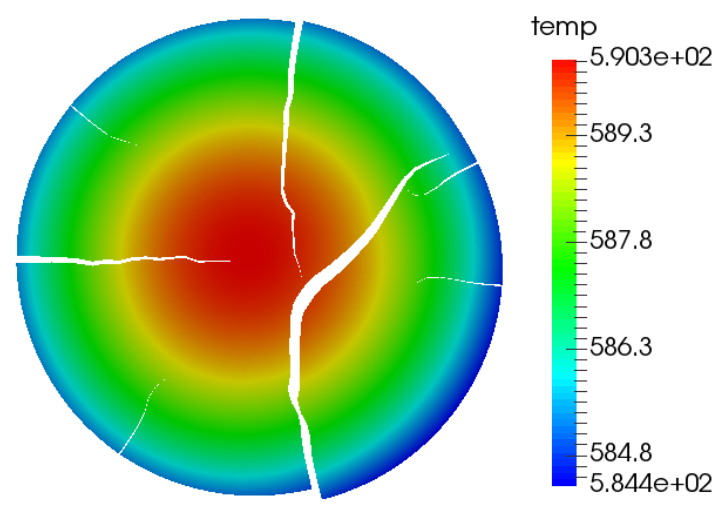

(d) $\mathrm{t}=40000 \mathrm{~s}$

Figure 7: Temperature field from XFEM simulations with the residual opening model. Displacements are magnified 10x. Although the model had fuel and cladding, only the fuel is shown. 


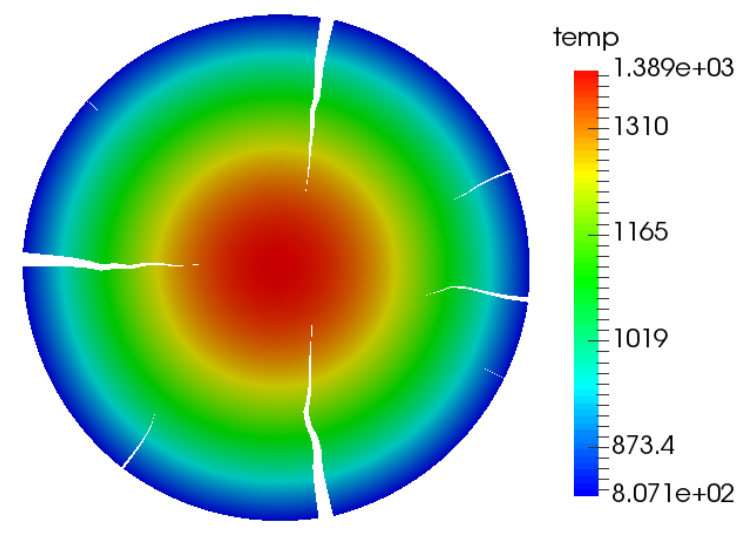

(a) $\mathrm{t}=10000 \mathrm{~s}$

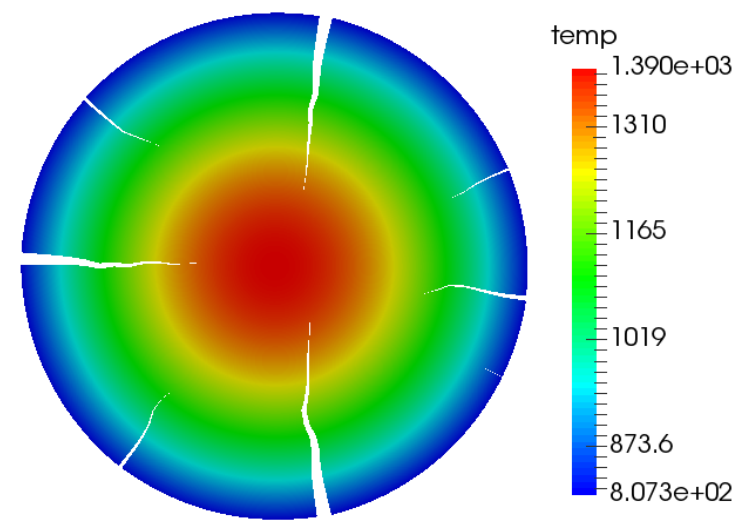

(c) $\mathrm{t}=30000 \mathrm{~s}$

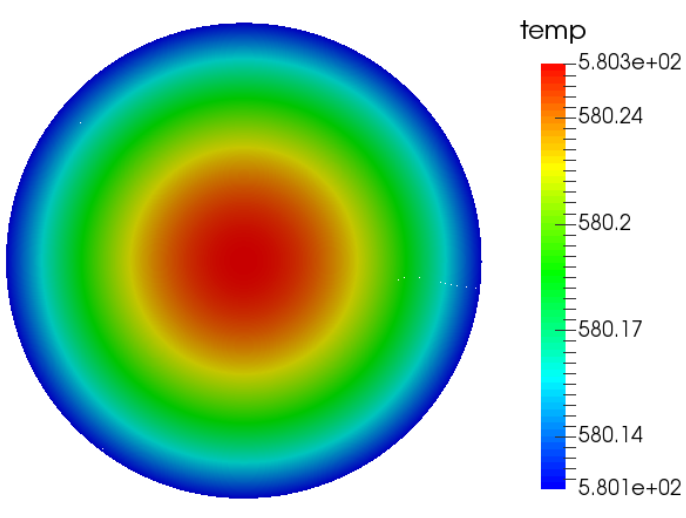

(b) $\mathrm{t}=20000 \mathrm{~s}$

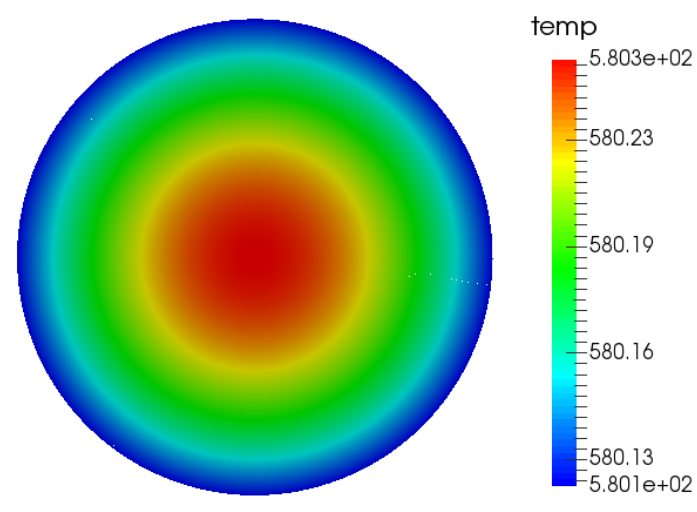

(d) $\mathrm{t}=40000 \mathrm{~s}$

Figure 8: Temperature field from XFEM simulations without the residual opening model. Displacements are magnified 10x. Although the model had fuel and cladding, only the fuel is shown. 


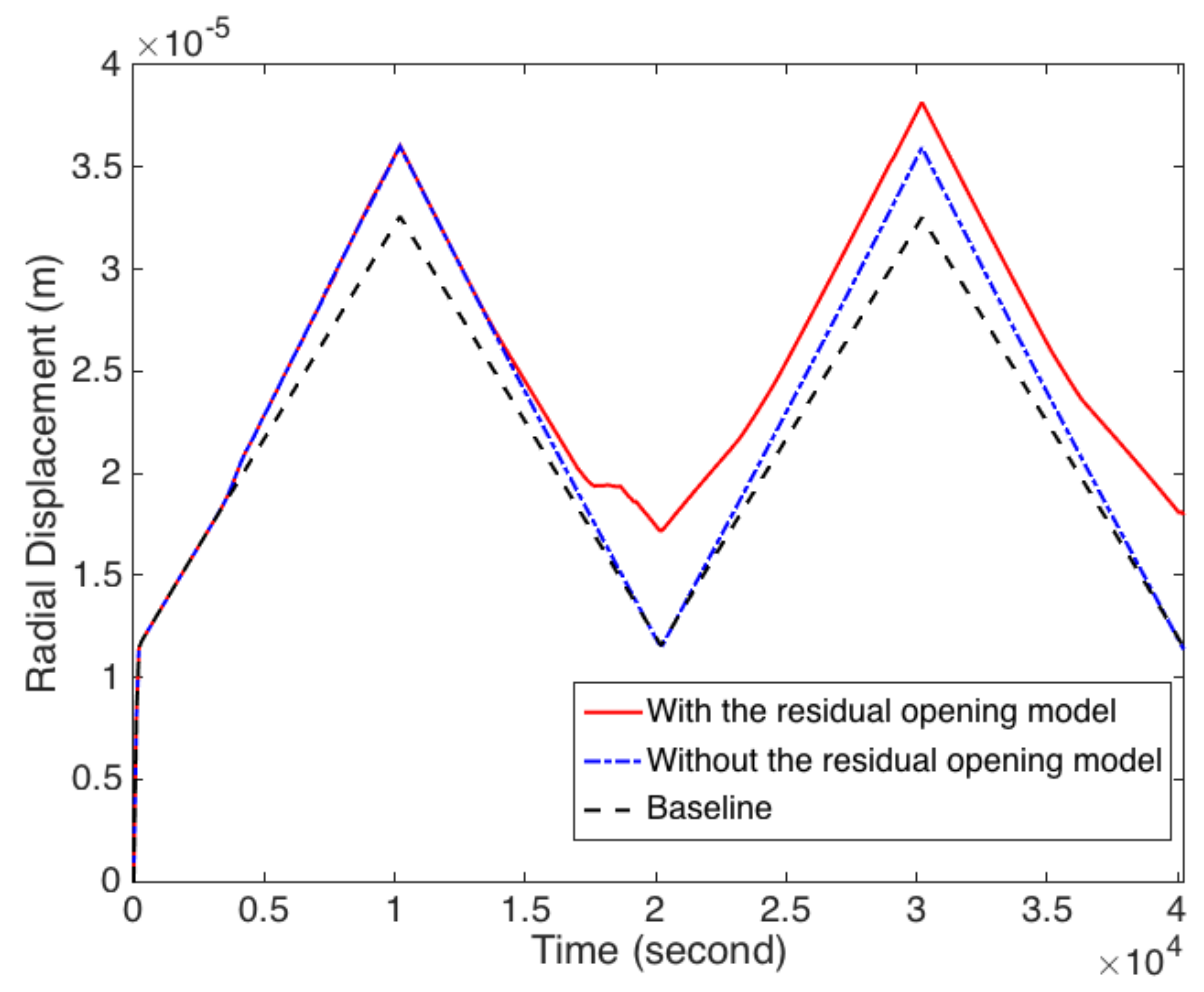

Figure 9: Comparison of radial displacement predicted with and without the residual opening model, as well as for a baseline case without fracture. 


\section{Summary and Future Work}

A number of improvements have been made to improve the ability to realistically model the physical mechanisms underlying relocation phenomena in LWR fuel using XFEM with the BISON fuel performance code:

- A system to permit use of stateful material properties for interaction models between the opposing surfaces of a crack modeled with XFEM has been developed. This permits the use of a model that includes the effects of residual opening across that interface.

- The system for evaluating fracture integrals in MOOSE has been modified to allow its use with an evolving set of crack tips and crack front points. With some additional development, this will be used to provide a criterion for crack growth within XFEM.

- Integrated boundary conditions can now be correctly imposed on surfaces intersected by fractures represented using XFEM. This improves the accuracy of LWR fuel models using XFEM that include both the fuel and the cladding.

These improved capabilities have permitted the analysis of relocation due to fracture including the effects of residual opening. The residual opening results in increased radial relocation after power cycles, and causes radial cracks to propagate in a circumferential direction during a power-down.

The capabilities for crack propagation using XFEM demonstrated here provide a very useful foundation for physicsbased modeling of relocation, which will improve the ability of BISON to be a predictive tool under a wider variety of conditions than it currently is.

The model used here for interaction of cracks is a simplified representation of actual behavior. Developing a model based on experimental observations to include a more physically-based representation of interface behavior is an important area of future work. In addition, these models should be validated against experimental data. Finally, although the crack propagation models shown here are quite powerful, there are still some important limitations. There is limited ability to handle crack branching and coalescence, and crack propagation in $3 \mathrm{D}$ is not yet supported. These are all capabilities that are very feasible to be added to this code with more development effort. 


\section{References}

[1] Eds. M. A. Kramman, H. R. Freeburn. ESCORE-the EPRI steady-state core reload evaluator code: General description. Technical Report EPRI NP-5100, Electric Power Research Institute, February 1987.

[2] Laura P. Swiler, Richard L. Williamson, and Danielle M. Perez. Calibration of a fuel relocation model in BISON. In International Conference on Mathematics and Computational Methods Applied to Nuclear Science \& Engineering, Sun Valley, Idaho, May 5-9 2013. American Nuclear Society.

[3] B. W. Spencer, W. Jiang, H. Chen, Y. Hu, C. Peco, and J. E. Dolbow. Evaluation of relocation effects modeled using discrete fracture models, CASL FY16 letter report. Technical report, Idaho National Laboratory, 2016.

[4] Benjamin Spencer, Marie Backman, Pritam Chakraborty, and William Hoffman. Reactor pressure vessel fracture analysis capabilities in Grizzly. Technical Report INL/EXT-15-34736, Idaho National Laboratory, Idaho Falls, ID, March 2015.

[5] John Dolbow, Ziyu Zhang, Benjamin Spencer, and Wen Jiang. Fracture capabilities in Grizzly with the eXtended Finite Element Method (X-FEM). Technical Report INL/EXT-15-36752, Idaho National Laboratory, Idaho Falls, ID, September 2015.

[6] Ziyu Zhang, Wen Jiang, John E. Dolbow, and Benjamin W. Spencer. A modified moment-fitted integration scheme for X-FEM applications with history-dependent material data. Computational Mechanics, accepted. 